\title{
Implicaciones en el movimiento humano de las adaptaciones del esquema e imagen corporal secundarias a una amputación
}

\author{
Implications in human movement of the adjustments about the scheme and body \\ image as an outcome of an amputation
}

Hugo Ricardo Reséndiz Vega ${ }^{*}$, Mónica Mariana Serrano Rosales.

\section{Resumen}

Introducción: el movimiento corporal humano es el resultado de la interacción entre elementos psicológicos, biológicos y sociales. Las amputaciones generan alteraciones en la estructura mental del individuo ocasionando restricciones en la participación social; por ello, es necesario comprender la influencia de la imagen y el esquema corporal de los sujetos con amputación según la teoría del movimiento continuo. Métodos: Se realizó una revisión de la literatura en las bases de datos PubMed, Science Direct, Clinical Key, PEDro, SciELO en el mes de abril de 2020; la selección de artículos se dividió en 3 fases, utilizando los términos "body schema", "body image", "amputee" $y$ "movement". Resultados: se encontró un total de 142 artículos, de los cuales se incluyeron 13 que cumplían con los criterios de selección. Discusión: posterior a una amputación se producen alteraciones de la imagen y el esquema corporal, además de cambios plásticos en el sistema nervioso central; derivado de esto existirán modificaciones en el movimiento corporal de la persona. Se requiere favorecer el manejo integral del sujeto con amputación basado en la teoría del movimiento continuo abarcando esferas psicológicas, biológicas y sociales. Conclusión: la aceptación e integración de la imagen y esquema corporal son fundamentales para restablecer la capacidad máxima de movimiento de la persona con amputación.

Palabras clave: esquema corporal; imagen corporal; amputación; movimiento.

\begin{abstract}
Introduction: Human body movement is the result of the interaction among psychological, biological and social elements; amputations generate alterations in the individual's mental structure causing restrictions on social participation; therefore, it is necessary to understand the influence of the image and body schema of amputee subjects according to the theory of continuous movement. Methods: A review of the literature was carried out in the databases PubMed, Science Direct, Clinical Key, PEDro, SciELO in April 2020; the selection of articles was divided into 3 phases, using the terms "body schema", "body image", "amputee" and "movement". Results: A total of 142 articles were found, of which 13 that met the selection criteria were included. Discussion: After an amputation, alterations of the image and body schema will occur. In addition to plastic changes in the central nervous system, therefore will be modifications in the person's body movement. It is necessary to favor the comprehensive management of the subject with amputation based on the theory of continuous movement, covering psychological, biological and social spheres. Conclusion: The acceptance and integration of the image and body schema are fundamental to reestablish the maximum capacity of movement of the person with amputation.
\end{abstract}

Keywords: body schema; body image; amputation; movement.

Fecha de envío: 4 de diciembre de 2019 - Fecha de aceptación: 24 de agosto de 2020

\section{Introducción}

La práctica de la fisioterapia está basada en el conocimiento del cuerpo humano incorporando información relevante de diferentes ciencias y disciplinas. Las deficiencias y la necesidad teórica para la enseñanza e investigación en fisioterapia llevaron al desarrollo de la teoría del movimiento continuo; el concepto clave de esta teoría es el movimiento. En esta, el fisioterapeuta conceptualiza

(1) Ciencias de la Rehabilitación en el Movimiento Humano, Facultad de Enfermería de la Universidad Autónoma de Querétaro, México.

*Autor de correspondencia: vegahugo19@gmail.com 
al movimiento como un continuo que incorpora aspectos físicos y patológicos del movimiento, con consideraciones sociales y psicológicas. De acuerdo a esta podemos decir que: 1) el movimiento es esencial para la vida humana, 2) el movimiento ocurre de niveles microscópicos a macroscópicos, 3) el movimiento está influenciado por factores físicos, psicológicos, sociales y ambientales, 4) los niveles del movimiento son interdependientes, 5) cada nivel del continuo de movimiento tiene un máximo potencial de movimiento alcanzable, que es influenciado por los otros niveles, 6) cada individuo tiene una capacidad de movimiento actual y una capacidad de movimiento preferido, 7) los factores patológicos tienen el potencial de crear grandes diferencias entre la capacidad máxima, actual y preferida de movimiento del individuo, 8) el objeto de la fisioterapia es minimizar las diferencias potenciales entre las 3 capacidades de movimiento (Cott et al., 1995). El modelo propuesto por la teoría del movimiento continuo se aprecia en la Figura 1.

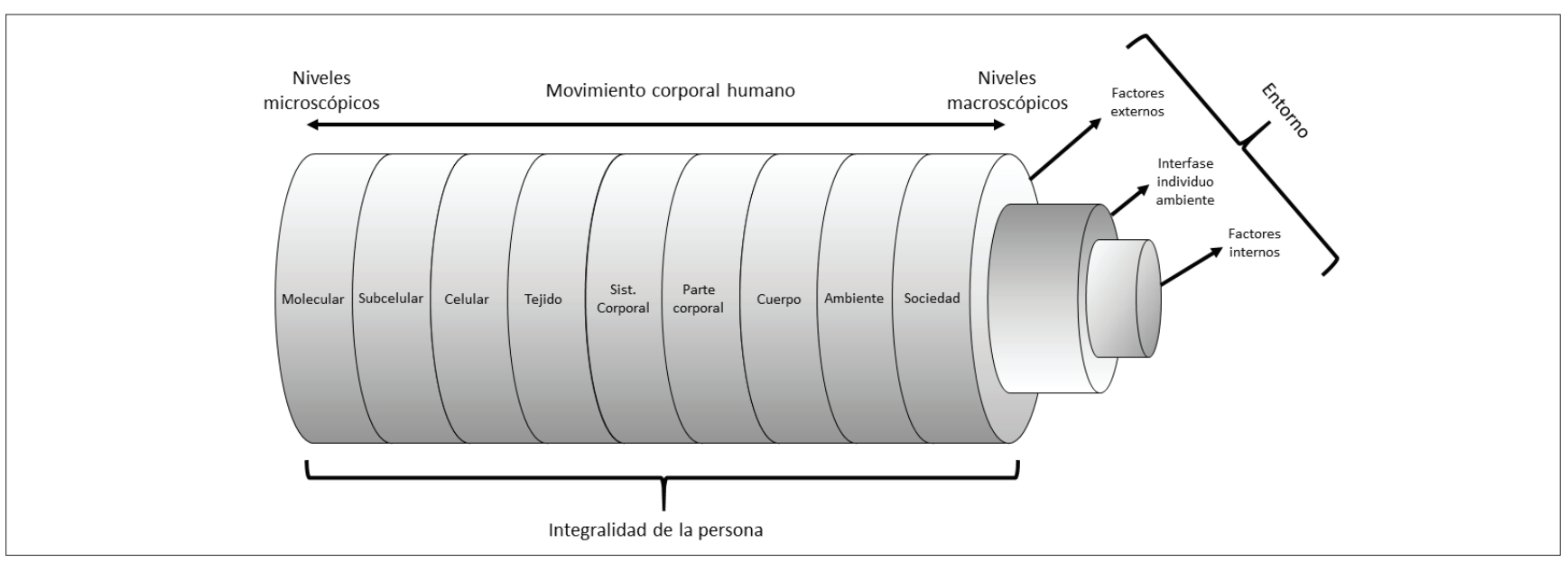

Figura 1: Modelo del movimiento corporal según la teoría del movimiento continuo.

En esta figura se detalla la interdependencia entre los niveles microscópicos (atómicos) y macroscópicos (sociales) que integran el movimiento corporal; siendo a la vez influenciado por factores intrínsecos, extrínsecos y el entorno del ser humano de acuerdo a lo establecido en la teoría del movimiento continuo.

De acuerdo con Torres (2014), el esquema corporal hace referencia un proceso de integración sensitiva y motora; en otras palabras, al conocimiento que tenemos los seres humanos de nuestro cuerpo; así como de la interpretación que damos al mismo en relación al entorno; de manera que, el esquema corporal se forma por el conocimiento espacial del cuerpo humano por medio de la información sensorial aferente $y$, por tanto, constituye un elemento primordial en la interacción con el entorno. Por lo que, acorde a la teoría del movimiento continuo, el esquema corporal es esencial para la vida, debido a que el movimiento corporal es una consecuencia del reconocimiento del mismo, que permitirá delimitar al yo y al entorno, pero a su vez, relacionarse entre ellos (Torres, 2014).

Por otro lado, la imagen corporal, es una mezcla percibida entre lo que se ve y se siente. El cuerpo imaginario que se visualiza se deforma con el tiempo con las experiencias agradables y desagradables que viven los seres humanos. Estas experiencias modifican poco a poco lo percibido frente a un espejo, manteniendo constante el sentimiento de la persona. Tras un evento traumático, se desarrolla una modificación temporal de esta imagen, eventualmente todo vuelve a la normalidad y se conserva la imagen corporal; sin embargo, existen situaciones donde la modificación de la imagen es permanente, adentrando al sujeto en un conflicto en el que no existe un reconocimiento de sí mismo; es capaz de verse en las demás personas, pero se visualiza a sí mismo de una manera fragmentada (Vega et al., 2011; De la Puerta Huertas, 2014; Meingüer et al., 2014).

Con base en lo anterior, tras una amputación se presentan diversas complicaciones neurológicas, motrices, sensitivas y físicas las cuales incrementan el grado de discapacidad restringiendo la movilidad, debido a esto se propone que el abordaje de los pacientes con amputación se realice de manera integral, en el cual se debe involucrar el control médico, la rehabilitación física, la evaluación y abordaje psicológico para finalizar con el proceso de fabricación y adaptación a una prótesis; esto apoya a la concepción establecida por la teoría del movimiento continúo (Vázquez, 2016).

En atención a esto, es necesario entender que para desarrollar el máximo del potencial de movimiento corporal después de una amputación no basta únicamente con el desarrollo de capacidades físicas; sino que, además, se requiere promover y facilitar la aceptación de la nueva configuración anatómica. De manera que, el presente artículo pretende responder a la pregunta ¿Cuál es la influencia de la imagen y el esquema corporal en el movimiento de personas con amputación? 


\section{Metodología}

Se realizó una revisión de la literatura en tres fases; durante la primera fase se realizó la búsqueda en las bases de datos PubMed, Science Direct, Clinical Key, PEDro, SciELO en el mes de abril de 2020. Los términos de búsqueda fueron "body schema", "body image", "amputatee" " "movement", la búsqueda se llevo a cabo con estos términos y/o sus sinónimos en idioma inglés o español, acompañados de los operadores booleanos "and", "y", "\&" y "+" para una búsqueda más rigurosa; los términos utilizados se definieron previamente en $\mathrm{MeSH}$.

Posteriormente, en una segunda fase se revisó título y resumen de los resultados obtenidos; se consideraron como criterios de inclusión aquellos artículos cuya población de estudio fueran amputados, las variables de estudio fueran el esquema o la imagen corporal y el movimiento; sin restricción temporal, únicamente artículos pertenecientes a revistas indexadas, en idioma inglés, español o francés y cualquier tipo de estudio. Se excluyeron aquellos artículos que fueran revisiones sistemáticas o meta-análisis y artículos donde la población estudiada no fueran sujetos con amputación.

En una tercera fase, se realizó la lectura de todos los artículos potencialmente elegibles y se agruparon de acuerdo a su tématica con los términos de búsqueda propuestos. Durante esta fase los artículos fueron sometidos a revisión por parte de dos revisores para considerar los artículos de la selección final. El flujo de estrategia de búsqueda se puede observar en el Figura 2.

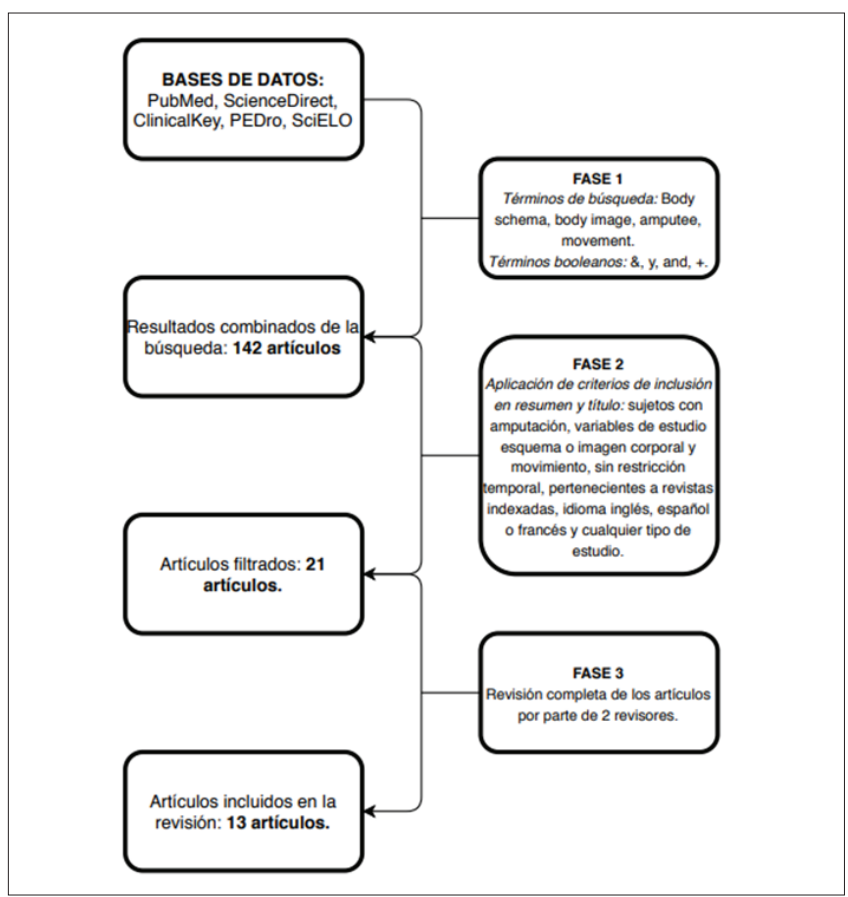

Figura 2: Diagrama de flujo de la estrategia de búsqueda. En esta figura se observan las 3 fases de la estrategia de búsqueda y los artículos seleccionados durante cada una de ellas.

\section{Resultados}

Durante la primera fase, la búsqueda arrojó 142 resultados, posterior a la aplicación de criterios de selección la muestra se redujo a 21 artículos; finalmente, en la revisión final por parte de los revisores se eliminaron 8 artículos debido a que; por un lado, el estudio hacía referencia a las amputaciones, imagen y esquema corporal, pero la población de estudio eran individuos sanos; y por el otro, eran artículos que se encontraban repetidos.

De los 13 artículos incluidos para la revisión, 11 (84,6\%) pertenecían a PubMed, en Science Direct y Clinical Key se encontró un artículo (7,7\% respectivamente) en cada base de datos; en el caso de las plataformas PEDro y SciELO, no se encontró ningún artículo vinculado a los criterios de búsqueda y selección. En la Tabla 1, se observan los resultados obtenidos durante la revisión.

\section{La diferencia entre el esquema corporal y la imagen corporal}

Fisher (1986) define la imagen corporal como la imagen de nuestro propio cuerpo. Refiere al cuerpo como una experiencia psicológica que incluye nuestras emociones y actitudes, desarrolladas a través de nuestra experiencia. Schilder (1935) ve la conciencia del cuerpo como una experiencia subjetiva del mismo; la complejidad de los principios del esquema corporal, la postura corporal y la posición del cuerpo en relación con los movimientos espaciales. Por otro lado, el esquema del cuerpo es una representación central de las características espaciales del cuerpo que incluyen la longitud de sus segmentos, su estructura jerárquica y configuración y la forma de las superficies del cuerpo. De manera que la imagen corporal es la representación visual consciente de cómo vemos nuestros cuerpos desde afuera y no está estrechamente relacionada con el control de movimiento. Haggard y Wolpert (2005) reconocen 7 características del esquema corporal: codificado espacialmente, modular, adaptable, supramodular, coherente, interpersonal y su papel en la actualización de los movimientos. Es así como el esquema del cuerpo representa la posición y la configuración del cuerpo como material expansivo en el espacio, cuya función principal es integrar la información táctil proveniente de las áreas del cuerpo y la información propioceptiva sobre las extremidades. En relación a lo anterior se puede decir que el esquema corporal se adapta a los cambios graduales de las características espaciales del cuerpo; por ejemplo, el uso de herramientas condiciona un cambio en la función de las neuronas cerebrales y en su sistema relacional. (Mayer et al., 2008). 
Tabla 1: Selección final de artículos incluidos en la revisión.

\begin{tabular}{|c|c|c|c|c|c|}
\hline $\begin{array}{l}\text { Base de } \\
\text { datos }\end{array}$ & Autores & Año & Título & Población & Tipo de estudio \\
\hline ClinicalKey & Arieta, A. et al. & 2002 & $\begin{array}{l}\text { A fMR } 1 \text { study of the cross modal interaction in } \\
\text { the brain with an adaptable EMG prosthetic hand } \\
\text { with biofeedback }\end{array}$ & $\begin{array}{l}1 \text { sujeto femenino con amputación de brazo } \\
\text { de } 50 \text { años de edad y } 2 \text { controles sin ninguna } \\
\text { alteración. }\end{array}$ & $\begin{array}{l}\text { Transversal } \\
\text { comparativo }\end{array}$ \\
\hline Pubmed & Akarsu, S. et al. & 2012 & $\begin{array}{l}\text { Quality of life and functionality after lower limb } \\
\text { amputations: comparision between uni-vs. Bilateral } \\
\text { amputee patients }\end{array}$ & $\begin{array}{l}15 \text { bilaterales y } 15 \text { amputados unlitaterales } \\
\text { de extremidad inferior que sirvieron en las } \\
\text { fuerzas armadas turcas. }\end{array}$ & $\begin{array}{l}\text { Transversal c } \\
\text { omparativo }\end{array}$ \\
\hline Pubmed & André, J.M. et al. & 2001 & $\begin{array}{l}\text { Classification et mécanismes des perceptions et } \\
\text { illusions corporelles des amputés }\end{array}$ & $\begin{array}{l}60 \text { sujetos con amputación de distintas } \\
\text { etiologías ( } 37 \text { traumáticas, } 23 \text { vasculares), } \\
\text { segmentos ( } 30 \text { de miembros superiores y } \\
30 \text { de miembros inferiores). }\end{array}$ & $\begin{array}{l}\text { Observacional Des- } \\
\text { criptivo Prospectivo }\end{array}$ \\
\hline Pubmed & Cuberovic, I. et al. & 2019 & $\begin{array}{l}\text { Learning of artificial sensation through long term } \\
\text { home use of a sensory enabled prothesis }\end{array}$ & $\begin{array}{l}1 \text { amputado transradial masculino de lado } \\
\text { derecho. }\end{array}$ & $\begin{array}{l}\text { Cuasiexperimental } \\
\text { Longitudinal Pros- } \\
\text { pectivo }\end{array}$ \\
\hline Pubmed & Desrochers, J. et al. & 2018 & $\begin{array}{l}\text { Relationship between body image and } \\
\text { physical functioning following rehabilitation for } \\
\text { lower limb amputation }\end{array}$ & $\begin{array}{l}30 \text { pacientes amputados de miembro inferior del } \\
\text { Amputee Rehabilitation Program. }\end{array}$ & $\begin{array}{l}\text { Cohorte } \\
\text { prospectivo }\end{array}$ \\
\hline Pubmed & Ferreira, L. et al. & 2018 & $\begin{array}{l}\text { Brazilian Portuguese version of the amputee } \\
\text { body image scale: Cultural adaptation and a } \\
\text { psychometric analysis }\end{array}$ & $\begin{array}{l}100 \text { adultos amputados de } 18-94 \text { años, el } 74 \% \\
\text { es amputado de miembro inferior y el } 24 \% \text { de } \\
\text { miembro superior, y un } 2 \% \text { fue amputación } \\
\text { de miembro superior e inferior. }\end{array}$ & $\begin{array}{l}\text { Observacional Trans- } \\
\text { versal } \\
\text { Descriptivo }\end{array}$ \\
\hline Pubmed & Gouzien, A. et al. & 2017 & $\begin{array}{l}\text { Reachability and the sense of embodiment in } \\
\text { amputees using prostheses }\end{array}$ & $\begin{array}{l}12 \text { pacientes con amputación de mano a } \\
\text { nivel de antebrazo y } 30 \text { sujetos sanos como } \\
\text { controles. }\end{array}$ & $\begin{array}{l}\text { Transversal com- } \\
\text { parativo }\end{array}$ \\
\hline Pubmed & Gozaydinoglu,S.etal. & 2019 & $\begin{array}{l}\text { Body image perception, compliance with a pros- } \\
\text { thesis and cognitive performance in transfemoral } \\
\text { amputees }\end{array}$ & $\begin{array}{l}45 \text { sujetos con amputación transfemoral } \\
\text { unilateral de causa traumática con una edad } \\
\text { media de } 18-50 \text { años. La edad media de la } \\
\text { amputación fue de } 12.6+-9,4 \text { años. }\end{array}$ & $\begin{array}{l}\text { ObservacionalTrans- } \\
\text { versal Descriptivo }\end{array}$ \\
\hline Pubmed & Lorimer, G. et al. & 2009 & $\begin{array}{l}\text { Interdependence of movement and anatomy } \\
\text { persists when amputees learn a physiologically } \\
\text { impossible movement of their phantom limb }\end{array}$ & $\begin{array}{l}7 \text { Sujetos con amputación de miembro superior } \\
\text { con síndrome de miembro fantasma en el brazo. }\end{array}$ & $\begin{array}{l}\text { Cuasiexperimental } \\
\text { Longitudinal Pros- } \\
\text { pectivo }\end{array}$ \\
\hline Pubmed & Mayer, A. et al. & 2008 & Body schema and body awareness of amputees & $\begin{array}{l}\text { Para configuración de esquema corporal: } 51 \\
\text { amputados distintos niveles de amputación. } \\
\text { Para el aspecto funcional del esquema corporal: } \\
34 \text { amputados. }\end{array}$ & $\begin{array}{l}\text { Transversal com- } \\
\text { parativo }\end{array}$ \\
\hline $\begin{array}{l}\text { ScienceDi- } \\
\text { rect }\end{array}$ & Nakagawa, K. et al. & 2019 & $\begin{array}{l}\text { Cortical reorganization of lower-limb motor } \\
\text { representations in an elite archery athlete with } \\
\text { congenital amputation of both arms. }\end{array}$ & $\begin{array}{l}\text { Arquero de } 35 \text { años de edad con amputación } \\
\text { congénita bilateral de miembro superior. Se } \\
\text { reclutaron a } 19 \text { sujetos sin amputación diestros. }\end{array}$ & $\begin{array}{l}\text { Observacional Trans- } \\
\text { versal Comparativo }\end{array}$ \\
\hline Pubmed & Raffin, E. et al. & 2016 & $\begin{array}{l}\text { Primary motor cortex changes after amputation } \\
\text { correlate with phantom limb pain and the ability } \\
\text { to move the phantom limb }\end{array}$ & $\begin{array}{l}11 \text { amputados de miembro superior y } 17 \\
\text { controles sanos. }\end{array}$ & $\begin{array}{l}\text { Transversal com- } \\
\text { parativo }\end{array}$ \\
\hline Pubmed & Wetterhahn, K. etal. & 2002 & $\begin{array}{l}\text { Effect of participation in physical activity on body } \\
\text { image of amputees }\end{array}$ & $\begin{array}{l}56 \text { participantes con amputación de miembro } \\
\text { inferior de entre } 20 \text { y } 89 \text { años de edad. }\end{array}$ & $\begin{array}{l}\text { Observacional de } \\
\text { Asociación Trans- } \\
\text { versal }\end{array}$ \\
\hline
\end{tabular}




\section{El esquema corporal y las reorganizaciones corticales en sujetos con amputación}

Actualmente, se sabe que la información propioceptiva forma un papel importante en la disposición del esquema corporal; además, se ha analizado la conexión entre el sistema vestibular y el esquema corporal, es por ello que, el estímulo vestibular es fundamental en la construcción del esquema corporal (Mayer et al., 2008). La reorganización cortical después de perder los estímulos de la extremidad amputada, se debe a la modalidad cruzada; es decir, recibe señales de entrada de neuronas adyacentes, lo que resulta en lo que se denomina como "extremidad fantasma". Como se mencionó anteriormente; cuando se proporcionan estímulos simultáneos, el cerebro puede asociar los estímulos en un evento único; por lo que, es posible decir, que es se puede forzar al cerebro a producir nuevas sensaciones, siempre que el estímulo sea simultáneo; de tal manera, que los sujetos que usan una mano protésica pueda tener retroalimentación sensorial además de la visual (Hernández et al., 2006).

Recientemente, la somatosensación se ha restaurado en prótesis de miembros superiores mediante técnicas electrocutáneas y vibrotáctiles no invasivas o mediante interfaces neuronales implantadas. Esto ha llevado a múltiples grupos a investigar la estimulación eléctrica directa de los nervios restantes para restaurar la sensación de la mano faltante (Cuberovic et al., 2019). Por ejemplo, se ha medido la activación en la región somatosensorial S1 del cerebro para ver los efectos de la estimulación eléctrica cuando funciona como biofeedback en manos protésicas mioeléctricas, utilizando el movimiento de flexión/extensión de los dedos para agarrar una esfera con la mano protésica, agarrando la esfera tan pronto la viera acercarse a la prótesis, esto activaba sensores de presión colocados en la mano y la presión se traducía en una señal de estimulación eléctrica transcutánea. Los resultados demostraron un área de activación más pequeña en la corteza motora cuando se usa la estimulación eléctrica que cuando sólo se tiene una retroalimentación visual. Por otro lado, se observó que la estimulación eléctrica no se detectaba en la región $\mathrm{S} 1$, sino que se propaga en el lóbulo parietal, a cargo del procesamiento de información somatosensorial. La prueba muestra que existe una reorganización cortical; esto lleva a pensar que, para los sujetos con amputación, la activación en el cerebro aumenta a lo largo de la corteza motora primaria. (Hernández et al., 2006).

La explicación a esto yace en el aprendizaje perceptivo, ya que este, está habilitado por la plasticidad de la corteza somatosensorial e implica aumentos en el tamaño de la representación de un estímulo en la corteza somatosensorial, el estrechamiento de la selectividad de las células sintonizadas, y cambios en las relaciones temporales de las neuronas. Es importante considerar que la plasticidad somatosensorial del adulto es un factor clave de recuperación, debido a que los cursos de tiempos asociados con la reorganización cortical pueden variar desde semanas hasta un año. Aunado a esto, la realización de una prótesis tiene resultados psicosociales importantes, tales como la percepción consciente de la herramienta dentro de los límites corporales y el procesamiento sensoriomotor de la herramienta como si perteneciera al cuerpo. Es así que la colocación de una herramienta altera el procesamiento de eventos sensoriales en el espacio peripersonal y personal y puede modificar el esquema corporal. Por ejemplo, la implementación de una prótesis sensorial a un sujeto con amputación durante 49 días arrojó un incremento en puntajes de autoeficiencia, la imagen corporal, el contacto social y la eficiencia de la prótesis, esto cuando se usaba la retroalimentación sensorial. A medida que el sujeto aprendió la somatosensación artificial, refinó su capacidad de integrar la sensación artificial en su representación corporal existente, lo que condujo a acciones protésicas más eficientes y precisas, además de una disminución en la atención requerida para usar la prótesis; esto parece indicar la incorporación de la prótesis al esquema corporal, ya que el espacio peripersonal tiene prioridad en la atención. Por lo que, este tipo de cambios sensoriales probablemente fueron resultado de un aprendizaje activo y pasivo, lo que sugiere que la plasticidad cortical puede mediar el aprendizaje sensorial incluso para sensaciones artificiales producidas por estimulación eléctrica (Cuberovic et al., 2019). Dicho en otras palabras, se sabe que en un sujeto sin amputación se ha demostrado que el uso de un rastrillo durante unos minutos extiende el espacio peripersonal alrededor de la mano que lo sostiene y modifica el tamaño percibido de la mano que se percibe como más larga. Unos minutos después de que se interrumpe el uso de la herramienta, los campos receptivos visuales vuelven a su tamaño original; en amputados de extremidad superior los límites del espacio peripersonal se extienden igualmente mediante el uso de una prótesis (Gouzien et al., 2017).

En relación a lo último, se ha comparado a sujetos con amputación de la extremidad superior contra sujetos sanos para que juzgaran si podían alcanzar un objeto que se les presentaba; ya que, esta tarea requiere estimar visualmente la distancia del objetivo, explotando el conocimiento corporal sobre la longitud funcional del brazo, y el conocimiento motor sobre la factibilidad de la acción en el contexto específico. Destaca la aparición de 3 resultados principales. Primero: el espacio de alcance real en sujetos con amputación era más pequeño con su prótesis que con su miembro sano; sin embargo, juzgaron que podían llegar tan lejos con su prótesis como con su miembro sano. Segundo: utilizando la extremidad sana, los sujetos con amputación sobreestimaron más el alcance de su espacio de alcance que los sujetos sanos. 
Finalmente, su sobreestimación parece ser menor con la prótesis que con la extremidad sana cuando la integración de la prótesis fue muy buena. Es importante comprender las capacidades motoras reales con el uso de una prótesis, para estudiar la representación que los sujetos amputados tienen de su espacio de alcance. Por ejemplo, en este caso la capacidad de alcance para los sujetos con amputación era aproximadamente $5 \mathrm{~cm}$ menor con su prótesis que con la mano sana, esto demuestra que la prótesis sólo restauró parcialmente la capacidad física de la extremidad amputada para actuar a una gran distancia. La ejecución de movimientos por un sujeto con amputación de extremidad superior suele tener errores marcados porque están acostumbrados a hacer movimientos más grandes de tronco para compensar el deterioro de la extremidad superior. En el caso de los amputados de miembros inferiores con prótesis, se ha señalado que presentan algunas modificaciones de la coordinación del movimiento en el lado sano, posiblemente debido a mecanismos compensatorios del cuerpo para garantizar acciones simétricas; esto se debe a que se altera la percepción general que tienen los pacientes de la capacidad de acción de su cuerpo como un todo (Gouzien et al., 2017).

Otras investigaciones mencionan que, al observar las acciones realizadas por otra persona, las regiones del propio sistema sensoriomotor se activan, involucrando representaciones motoras corticales específicas que corresponden a las partes del cuerpo observadas. Esta resonancia motora entre las acciones observadas y la representación motora propia se produce en una región de redes en la circunvolución frontal inferior, la corteza premotora ventral y el lóbulo parietal inferior, colectivamente denominado red de observación de la acción. Se ha observado que podemos involucrar regiones motoras cuando observamos acciones más allá de nuestras propias capacidades, sin embargo, los estudios que han analizado esto, utilizan acciones o efectores que eran visualmente familiares para el observador; por ejemplo, los individuos demuestran una mayor actividad sensoriomotora al observar acciones que ya han realizado o visto antes. Incluso, el entrenamiento con imágenes motoras de miembros fantasmas en sujetos con amputación conduce a cambios en la percepción de la propiedad y las cualidades cinemáticas del miembro fantasma, lo que sugiere que incluso el entrenamiento mediante imágenes de un miembro inexistente puede afectar las representaciones sensoriomotoras de ese miembro (Liew et al., 2013).

Por otro lado, se habla de una plasticidad desadaptativa que supone una "toma de posesión" ejecutada por otras partes del cuerpo; este modelo postula que el dolor persistente se asocia con una estructura y función preservadas en un área reorganizada de las partes vecinas del cuerpo. De manera que, existen asimetrías claras entre los hemisferios de sujetos amputados de extremidades superiores; es importante proporcionar más apoyo para el modelo de plasticidad desadaptativa, debido a que el dolor no es el único correlato perceptivo de la reorganización posterior a la amputación. En relación a esto una investigación con 11 sujetos amputados de miembro superior y 17 controles sanos demostró que después de la amputación, la corteza sensoriomotora primaria humana sufre una reorganización masiva que involucra cambio tanto estructural como funcional en el área motora primaria (SM1) contralateral a la amputación. Se observó que al abrir y cerrar la mano fantasma se producía una actividad sustancial en la corteza motora primaria contralateral a la amputación, demostrando que los patrones de reorganización están asociados con los patrones de uso de las extremidades. Dado que la organización topológica de la corteza sensorial se altera después de la amputación, todos los estudios utilizan movimientos voluntarios para examinar la reorganización de la corteza motora e inevitablemente miden los cambios dentro de la corteza misma, así como el reflejo de la reorganización de la corteza sensorial (Raffin et al., 2016). Incluso para la reorganización cortical es importante considerar las actividades realizadas por el sujeto; por ejemplo, se ha comparado a un atleta paralímpico con amputación congénita bilateral de extremidades superiores que utiliza los miembros inferiores para las actividades de la vida diaria y la arquería, contra otros sujetos sanos como controles. Se utiliza resonancia magnética funcional para valorar la actividad de las extremidades pélvicas y la estimulación magnética transcraneal para los miembros superiores. En este caso, el deportista mostró una expansión de la representación de miembro inferior en la $\mathrm{SM} 1$, indicando áreas expandidas de neuronas corticomotoras que inervan a músculos de miembros inferiores, especialmente al realizar movimientos de ortejos y rodilla. y una expansión lateral de SM1 sobre las representaciones de miembro superior y tronco (Nakagawa et al., 2019).

Finalmente, es importante considerar la influencia del miembro fantasma en sujetos con amputación y su asociación a los cambios corticales. En un estudio prospectivo a 60 sujetos con amputación (37 de etiología traumática y 23 de etiología vascular, 30 en miembros superiores y 30 en miembros inferiores, 6 con amputación bilateral, 15 con focomelia o la amputación se realizó antes de los 6 meses de vida) se les aplicó un interrogatorio preciso de las sensaciones que habían experimentado en su cuerpo desde la amputación. El resultado arrojó 3 anormalidades perceptivas: 1) percepción de la situación real (ausencia de una extremidad), 2) percepción exacerbada de la extremidad amputada (sensación de miembro fantasma), 3) percepción de un cuerpo "normal" previo a la amputación (ilusión de normalidad corporal). Se identificaron diferencias significativas para la sensación de miembro fantasma 
alterada, miembro deforme, miembro normal y sensación de no haber perdido la extremidad entre el grupo de sujetos con amputación traumática y sujetos con amputación congénita. No se encontraron diferencias significativas respecto a la etiología de la amputación, vascular o traumática. En relación a la ilusión de normalidad, difiere en que para los sujetos de amputación vascular o traumática la "normalidad" es antes de la amputación, y para los participantes de amputación congénita la "normalidad" es un cuerpo malformado. Esta información es compatible con los datos ya encontrados en la literatura sobre la percepción de miembro fantasma, particularmente los que la relacionan con la plasticidad cerebral (André et al., 2001). Lo anterior, confirma que la sensación que tenemos de nuestro cuerpo puede ser modificada por mecanismos generados intrínseca y extrínsecamente, que los cambios en el esquema corporal debido al miembro fantasma condicionan nuestro movimiento corporal y que nuestras estructuras anatómicas están conectadas entre sí. La interdependencia entre forma y función persisten aun cuando el cerebro construye nuevos patrones de movimiento y representaciones corporales (Lorimer \& Brugger, 2009).

\section{La imagen corporal del sujeto con amputación}

Como ya se ha establecido, la imagen corporal es la representación visual consciente de la persona; para estudiar su influencia en sujetos con amputación se han utilizado diferentes instrumentos; por ejemplo, la Amputee Body Image Scale (ABIS) se validó en un estudio a 56 sujetos con amputación de miembro inferior, de los cuales 24 fueron clasificados como activos ( 2 horas de ejercicio aeróbico semanal en sesiones de 20 minutos) y 32 como mínimamente activos; comparando sus resultados con la escala Multidimensional Body Self Relations Questionnaire (MBSRQ). Con esto se determinó que existe una relación positiva entre la actividad física que realizan los sujetos con amputación y su imagen corporal. La correlación entre las 6 sub-escalas de la MBSRQ validó la aplicación de ABIS como una herramienta de evaluación para sujetos con amputación; sin embargo, no se encontró correlación entre las sub-escalas de MBSRQ (cognición y comportamiento, orientación a la salud y orientación a la enfermedad) con la escala ABIS (Wetterhahn et al., 2002). También se ha validado la traducción al portugués de la ABIS, comparándola con la Body Appreciation Scale (BAS) para generar futuras propuestas de investigación y se propone la comparación entre sujetos con amputación sedentarios o físicamente activos, debido a que existen diferencias significativas entre los factores: 1 ) interés por la apariencia, 2) prótesis y muñón, y 3) función y discapacidad. Pese a que ABIS y BAS evalúan diferentes dimensiones de actitud en la imagen corporal (apreciación corporal y sentimientos o creencias negativas, respectivamente), se esperaba que tuviesen cierto nivel de asociación; sin embargo, se ha observado que presentan correlaciones relativamente pequeñas, por lo que es poco probable que esto indique una validez discriminante cuestionable (Ferreira et al., 2018).

Con base en la evidencia anterior, ABIS ha sido utilizado para establecer relaciones entre la imagen corporal y el desempeño cognitivo de sujetos con amputación, asociándola con la Trinity Amputation and Prothesis Experience Scale (TAPES) y la Montreal Cognitive Assessment (MoCA); en ese sentido, TAPES se ha utilizado para evaluar la adaptación a la amputación (adaptación psicosocial, restricciones en la actividad y satisfacción con la prótesis); mientras que, la escala MoCA evalúa la capacidad cognitiva de los sujetos. Se ha demostrado que existe una relación estadísticamente significativa entre la percepción de la imagen corporal del sujeto y el desempeño cognitivo. La percepción de la imagen corporal tiene relación negativa con la escala $\operatorname{MoCA}(r=-0,550, p=<0,001)$; además, existe una relación positiva con la adaptación psicosocial, satisfacción con la prótesis y la escala $\operatorname{MoCA}(r=0,550, p=<0,001)$. Por lo que, se concluye que la satisfacción con la imagen corporal, adaptación psicosocial, falta de restricciones en las actividades y satisfacción con la prótesis se asocian positivamente con el desempeño cognitivo. De tal manera que se recomienda incluir estos parámetros en la evaluación fisioterapéutica inicial para mejorar la funcionalidad del sujeto, tanto en el uso de su prótesis, como de su reintegración a la sociedad (Gozaydinoglu et al., 2019).

\section{La influencia del esquema y la imagen corporal en el proceso de rehabilitación}

El éxito de la rehabilitación en sujetos con amputación de algún segmento, depende de varios factores; tales como, enfermedades vasculares, la edad, el uso de aditamentos para la marcha, número de amputaciones, el equilibrio. Por ejemplo, los cambios del esquema corporal se ven asociados al tiempo de amputación y al uso de prótesis, tanto en sujetos que no la han usado como en los que han hecho uso de ella; de manera que estos parecen estar delimitados por un corto período de tiempo desde la amputación, lo cual, no permite que el cerebro reorganice los impulsos provenientes de la periferia y cree un esquema corporal alterado. Se ha observado que el reordenamiento del esquema corporal en cuatro usuarios principiantes con el uso de prótesis que sufrían enfermedad vascular previo a la amputación, sólo podían completar la ubicación espacial fantasma con un eje desviado. En este caso, la alternancia del esquema corporal podría haber comenzado en el período anterior a la amputación, por que las partes dolorosas del cuerpo se usaron menos o se usó ayuda médica para caminar; además, la diferencia en la distribución del peso corporal refleja el nivel de adaptación funcional de la prótesis en el esquema corporal. De manera que la asimilación de la prótesis como un nuevo medio para 
cambiar la ubicación y posición corporal habla de una adaptación del esquema corporal; ya que, la prótesis se integra en los límites corporales existentes; de manera que, la capacidad de usar una prótesis correctamente es fundamental; para ello, es importante incrementar la propiocepción del segmento (Mayer et al., 2008).

El proceso de rehabilitación impone demandas físicas cognitivas y psicosociales a los sujetos con amputación; en el caso de los sujetos con amputación de extremidad pélvica involucra la restauración de la movilidad independiente y mejora la calidad de vida. Lo más importante para la calidad de vida es la movilidad exitosa, los primeros meses después del alta son importantes para evaluar los factores que influyen en el aumento de movilidad, ya que la movilidad mejora hasta los 6 meses posteriores a la rehabilitación y luego se estabiliza (Desrochers et al., 2018). En relación a lo anterior; se sabe que las amputaciones traumáticas presentan problemas psicológicos y sociales, respecto a imagen corporal, actividades de autocuidado, movilidad y actividades ocupacionales y no ocupacionales. El estudio de 15 amputados bilaterales y 15 amputados unilaterales de extremidad inferior que sirvieron en las fuerzas armadas turcas mediante el cuestionario SF-36, el cuestionario de satisfacción de la prótesis, la escala de la imagen corporal del amputado, la escala de Houghton, la prueba de caminata de 6 minutos y el test de caminata de 10 metros mostró que los amputados unilaterales presentan un puntaje mayor en el SF36 en comparación a los sujetos amputados bilaterales; evidenciando una mejor calidad de vida en el caso de los primeros. Además, se observó que existe una correlación positiva entre los puntajes del SF-36 y la frecuencia de uso de prótesis, facilitando la movilidad de los sujetos participantes; sin embargo, existen varios factores como el confort, apariencia, peso y utilidad que son importantes para el uso de la prótesis; de manera que es importante que sean considerados; ya que esta, provee calidad de vida y proporciona imagen corporal normal, lo cual trae consigo un incremento de las capacidades físicas. Es así que, las bases del programa de rehabilitación en sujetos amputados deben comprender la recuperación de la mejor funcionalidad y movilidad locomotora posible, para ello es necesario evaluar la importancia del esquema y la imagen corporal durante todo el período de rehabilitación del paciente (Akarsu et al., 2012).

Por otro lado, la percepción de la imagen corporal tiene el potencial de impactar en la motivación y el compromiso social después de la amputación. Por ejemplo, se sabe que los veteranos de guerra con amputación de una extremidad pélvica que eran conscientes de su pierna protésica han demostrado una baja aceptación de su apariencia, lo que condiciona una reducción en la participación de actividades que antes disfrutaban, esto puede afectar directamente las ganancias de la movilidad funcional. Es necesario explorar la relación entre la percepción de la imagen corporal y la movilidad para optimizar la fase temprana posterior a la rehabilitación. Investigaciones en 30 pacientes amputados de extremidad pélvica del Amputee Rehabilitation Program entre abril 2016 y abril 2017 realizaron evaluaciones 48 horas después del alta y a los 4 meses en consulta médica subsecuente. Entre ambas mediciones, el puntaje medio en la escala Amputee Body Image Scale incrementó, indicando un nivel moderado de insatisfacción, demostrando una disminución en la percepción de la imagen corporal. Contrario a esto, los puntajes de movilidad mejoraron significativamente, y no se encontró correlación entre la valoración de la marcha y la imagen corporal. En este estudio se demuestra que la percepción de la imagen corporal no influye en los cambios de funcionamiento físico entre el alta y los 4 meses posteriores a la rehabilitación. Por lo que se piensa que el aumento significativo en la movilidad no está influenciado por la imagen corporal; sin embargo, el aumento de la actividad física realizada regularmente, conduce a una mejor percepción de la imagen corporal; de manera que, destaca la importancia de la actividad para promover una imagen corporal positiva (Desrochers et al., 2018).

\section{Conclusiones}

En conclusión, el esquema corporal es la representación central de las características espaciales del cuerpo y está íntimamente relacionado con el movimiento corporal humano. Su desarrollo depende de la información propioceptiva; tras un proceso de amputación existe una reorganización cortical que modifica el esquema corporal como una respuesta adaptativa; de manera que, la enseñanza de información propioceptiva artificial por medio de prótesis puede estimular la corteza somatosensitiva y a largo plazo mejorar las acciones de uso del dispositivo protésico y disminuir la atención que se presta para su uso; debido a que existe una integración de la prótesis al espacio peripersonal. Por otro lado, la imagen corporal es el resultado de las emociones y actitudes; el aumento significativo en la movilidad no está influenciado por la imagen corporal; contrario a esto, el incremento de la actividad física conduce a una mejor percepción de la imagen corporal. Finalmente, en el ámbito de la fisioterapia es importante favorecer el desarrollo de un esquema corporal adecuado para potencializar la capacidad de movimiento en los sujetos con amputación, el incremento en la movilidad promoverá una imagen corporal positiva, y la vez, esto facilitará la reintegración social.

\section{Fuentes de financiamiento}

No fue necesario ningún recurso financiero. 


\section{Contribuciones y conflictos declarados por los autores}

Reséndiz Vega y Serrano Rosales concibieron la idea, realizaron la búsqueda bibliográfica, redactaron el manuscrito y revisaron diferentes versiones del mismo. Los autores declaran no tener ningún conflicto de interés al realizar esta investigación.

\section{Referencias bibliográficas}

Akarsu S, Tekin L, Safaz I, Salim A. \& Yazicioglu K. (2012). Quality of life and functionality after lower limb amputations: comparison between uni-vs bilateral amputee patients. Prosthetics and Orthotics International 37, 9-13.

André JM, Paysant J, Martinet N. \& Beis JM. (2001). Classification et mécanismes des perceptions et ilusions corporelles des amputés. Ann Réadaptation Méd Phys 44, 13-8.

Cott C, Finch E, Gasner D. Yoshida K, Thomas S. \& Verrier M. (1995). The movement continuum theory of physical therapy. Physiotherapy Canada 47, 87-95.

Cuberovic I., Gill A, Resnik L, Tyler D. \& Graczyk E. (2019). Learning of artificial sensation through long term home use of a sensory enabled prosthesis. Front Neurosci 13.

De la Puerta Huertas R. (2014). Neuroplasticidad asociada a miembro fantasma. Rev Soc Esp del Dolor 21, 345-350.

Desrochers J, Frengopoulos C, Payne M., Viana, R. \& Hunter S. (2018). Relationship between body image and physical functioning following rehabilitation for lower limb-amputation. International Journal of Rehabilitation Research 42, 85-88.

Ferreira L, Meregui A, Mainenti M, Vigário P. \& Neves A. (2018). Brazilian portuguese version of the amputee body imagen scale: cultural adaptation and a psychometric analysis. Perceptual and Motor Skills 0, 1-18.

Gouzien A, de Vignemont F, Touillet A, Martinez N, De Graaf J, Jarrassé N. \& Roby-Brami A. (2017). Reachability and the sense of embodiment in amputees using prostheses. Scientific Reports $\mathbf{7}$.

Gozaydinoglu S, Hosbay Z., \& Durmaz, H. (2019). Body image perception, compliance with a prosthesis and cognitive performance in transfemoral amputees. Acta Orthopaedica et Traumatologica Turcica, 53, 221-225.

Hernández Arieta A, Kato R, Yokoi H. \& Arai T. (2006). A fMRI study of the cross-modal interaction in the brain with an adaptable EMG prosthetic hand with biofeedback. Annual International Conference, 1280-1284.
Liew S, Sheng T. \& Aziz-Zadeh L. (2013). Experience with an amputee modulates one's own sensoriomotor response during action observation. Neuroimage 69, 138-145.

Lorimer M. \& Brugger P. (2009). Interdependence of movement and anatomy persists when amputees learn a physiologically impossible movement of their phantom limb. PNAS 106, 18798.

Mayer A, Kudar K, Bretz K. \& Tihanyi J. (2008). Body schema and body awareness of amputees. Prosthetics and Orthotics International 32, 363-382.

Meingüer Cuevas Md, Martínez Tlahuel JL, Cuellar Hübe M, Galindo Vázquez Ó. \& Rojas Castillo E. (2014). Aspectos psicológicos en el paciente amputado: artículo de revisión. Tesis, Instituto Nacional de Cancerología, Servicio de Psicooncología, D.F. Accedido en http://incan-mexico.org/incan/docs/tesis/2014/altaespecialidad/Art.\%20psc\%20Meing\%C3\%BCer\%20Cuevas.pdf el 3 de diciembre de 2019

Mulvey MR, Fawkner HJ, Radford H. \& Johnson MI. (2009). The use of transcutaneous electrical nerve stimulation (TENS) to aid perceptual embodiment of prosthetic limbs. Medical Hypotheses, 72, 140-142.

Nakagawa K, Takemi M, Nakanishi T, Sasaki A. \& Nakazawa K. (2019). Cortical reorganization of lower-limb motor representations in an elite archery athete with congenital amputation of both arms. Neurolmage Clinical, 25

Raffin E, Richard N, Giraux P. \& Reilly K. (2016). Primary motor cortex changes after amputation correlate with phantom limb pain and the ability to move the phantom limb. Neuroimage, 130, 134-144.

Torres, G. (2014). Esquema, imagen, conciencia y representación corporal: mirada desde el movimiento corporal humano. Revista CES Movimiento y Salud 2, 80-88.

Vázquez Vela Sánchez E. (2016). Los amputados y su rehabilitación: Un reto para el estado, primera edición. Academia Nacional de Medicina de México. Intersistemas S.A. de C.V.

Vega V. de Vida P. \& Roitman D. (2011). Narcisismo e identificación en la fase del espejo: Una articulación entre Freud y Lacan. Universidad de Buenos Aires, Psicología evolutiva adolescencia, Buenos Aires. Accedido en https://docplayer.es/114789267-Narcisismo-e-identificacion-en-la-fase-del-espejo.html el 3 de diciembre de 2019.

Wetterhahn KA, Hanson C. \& Levy CE. (2002). Effect of participation in physical activity on body image of amputees. Am J Phys Med Rehabil 81, 194-201. 